Tohoku J. exp. Med., 1975, 117, 111-118

\title{
Experimental Study of Dibutyryl Cyclic AMP; Its Metabolic Effects Observed in Anesthetized Human Subjects
}

\author{
IKƯo SUEMoRI \\ Department of Anesthesiology,* School of Medicine, Kagoshima \\ University, Kagoshima
}

Stemori, I. Experimental Study of Dibutyryl Cyclic AMP; Its Metabolic Effects Observed in Anesthetized Human Subjects. Tohoku J. exp. Med., 1975, 117 (2), 111-118 Metabolic effects of $\mathrm{N}^{6}, \mathrm{O}^{2}$-dibutyryl adenosine $3^{\prime}, 5^{\prime}$ monophosphate (DBcAMP) were studied in 10 anesthetized patients who were divided at random into two groups each consisting of 5 patients. DBcAMP dissolved in $200 \mathrm{ml}$ of physiological saline was administered intravenously at a rate of $10 \mathrm{mg} / \mathrm{min}$ for $20 \mathrm{~min}$ in one group and $20 \mathrm{mg} / \mathrm{min}$ for $20 \mathrm{~min}$ in the other group. DBcAMP infusion at either rate increased levels of blood glucose, immunoreactive plasma insulin, blood pyruvate and blood redox-potential while it reduced levels of glycerol, non-esterified fatty acid and inorganic phosphate. These findings suggest that 200 and $400 \mathrm{mg}$ of DBcAMP stimulates glycogenolysis and glycolysis but inhibits lipolysis in man. - DBcAMP; glycogenolysis; glycolysis; lipolysis

Since adenosine $3^{\prime}, 5^{\prime}$-monophosphate (cAMP) was discovered as an intracellular mediator of glycogenolysis induced by epinephrine and glucagon in the liver by Sutherland and Rall (1958), it has been generally accepted that this nucleotide was a second messenger mediating a variety of hormonal effects at each target organ. Exogenous cAMP, however, was found to be impermeable in all cell membranes except for liver (Levine et al. 1969), though there is still some controversy (Heersche et al. 1971; Fujii and Okuda 1972). Although dibutyryl cyclic AMP, one of the cAMP derivatives, can penetrate the cell membrane more readily than cAMP does (Henion et al. 1967) and has almost the same biochemical properties in animal experiments, little is known on the metabolic and pharmacological responses of man to DBcAMP. In this study, metabolic effects of DBcAMP were investigated in anesthetized human subjects.

\section{MeThods}

Ten adult subjects weighing 42-68 $\mathrm{kg}$ who were to undergo a variety of operative procedures were chosen. Patients with any abnormal metabolic condition in preoperative

Received for publication, April 28, 1975.

* Director: Prof. J. Yoshitake.

Presented at the Annual Meeting of the Kyushu Society of Anesthesiology, November $10,1974$.

The following abbreviations are used: cAMP, adenosine $3^{\prime}, 5^{\prime}$-monophosphate; DBcAMP, $\mathrm{N}^{8}, \mathrm{O}^{2}$-dibutyryl cAMP; NEFA, nonesterified fatty acid; $\mathrm{Pi}$, inorganic phosphorus. 
TaBle 1. Description of subjects

\begin{tabular}{|c|c|c|c|c|c|}
\hline & Sex & Age & $\begin{array}{l}\text { Weight } \\
(\mathrm{kg})\end{array}$ & Diagnosis & Operation \\
\hline \multicolumn{6}{|c|}{ DBcAMP $200 \mathrm{mg}$ infusion group } \\
\hline M.Y. & $\mathrm{F}$ & 36 & 46 & Cervical cancer & Hysterectomy \\
\hline U.T. & $\mathrm{F}$ & 58 & 43 & Cervical cancer & Hysterectomy \\
\hline K.T. & $\mathrm{M}$ & 25 & 68 & Buerger's disease & Sympathectomy \\
\hline H.S. & $\mathrm{M}$ & 17 & 65 & Buerger's disease & Sympathectomy \\
\hline A.R. & $\mathrm{F}$ & 32 & 52 & Breast cancer & Mastectomy \\
\hline \multicolumn{6}{|c|}{ DBcAMP $400 \mathrm{mg}$ infusion group } \\
\hline M.A. & $\mathrm{F}$ & 44 & 42 & Uterine myoma & Hysterectomy \\
\hline T.K. & $\mathrm{F}$ & 20 & 46 & Uterine myoma & Hysterectomy \\
\hline S.S. & M & 49 & 57 & Gastric cancer & Gastrectomy \\
\hline Y.H. & $\mathrm{F}$ & 55 & 56 & Breast cancer & Mastectomy \\
\hline H.F. & $\mathrm{F}$ & 43 & 49 & Uterine myoma & Hysterectomy \\
\hline
\end{tabular}

examination were excluded. Premedication consisting of atropine sulfate (0.3-0.5 mg) and diazepam (10 mg.) was given intramuscularly $30 \mathrm{~min}$ before induction of anesthesia. Anesthesia was induced with nitrous oxide, oxygen and halothane in a semi-closed breathing system. Intubation was achieved with the aid of succinylcholine $(0.8 \mathrm{mg} / \mathrm{kg})$. Anesthesia was maintained with $\mathrm{N}_{2} \mathrm{O}-\mathrm{O}_{2}(3: 2 \mathrm{liter} / \mathrm{min})$ and less than 1 per cent halothane. Ventilation was controlled with an Ohio anesthesia ventilator to maintain $\mathrm{Pa}_{\mathrm{CO}_{2}}$ within normal range. Pancuronium bromide was given to secure respiratory paralysis. 10 patients were devided into two groups of the same number. 200 or $400 \mathrm{mg}$ of DBcAMP dissolved in $200 \mathrm{ml}$ of physiological saline solution were infused intravenously for a period of $20 \mathrm{~min}$ followed by infusion of physiological saline at a rate of $5 \mathrm{ml} / \mathrm{kg} / \mathrm{hr}$ during a period of observation. Blood samples were obtained through an indwelling catheter in the brachial vein before the start of infusion of DBcAMP, and additionally at $20 \mathrm{~min}$ intervals thereafter. Arterial blood was also drawn by arterial puncture to determine acid-base parameters. The blood glucose level was measured by the glucose oxidase method (Glucostat), plasma insulin by the method of Hales and Randle (1963), blood lactate, pyruvate and plasma glycerol enzymatically by biochemica test combination (Bergmeyer 1963), plasma NEFA by the Kram's method, plasma inorganic phosphate by the Fiske and Subbarow's method (1925), serum sodium and potassium by flame photometric analysis, serum chloride by the chloride meter (Corning, EEL.), the colorimetric estimation of calcium in serum by the o'cresolphthalein complexone method, blood redoxpotential by the platinum calomel electrode PK 149 previously described by Yoshimura et al. (1971), and arterial acid-base status by the Astrup's microequilibration technique (1957). DBcAMP as sodium salt was supplied by Daiichi Pharmaceutical Co., Ltd. Japan.

\section{Results}

DBcAMP infusion caused a significant elevation of blood glucose level, which reached the maximum between 20 and $40 \mathrm{~min}$ after termination of infusion in both groups (Fig. 1). The maximum blood glucose level was slightly higher but not significant with the higher doses of DBcAMP $(197 \mathrm{mg} / 100 \mathrm{ml}$ following $400 \mathrm{mg}$ infusion and $189 \mathrm{mg} / 100 \mathrm{ml}$ following $200 \mathrm{mg}$ infusion). Then the blood glucose level started to fall, but did not reach the preinfusion level even 120 min after infusion. Plasma insulin (IRI) rose sharply by the DBcAMP infusion and reached the maximum level at the end of infusion in both groups, which declined somewhat 


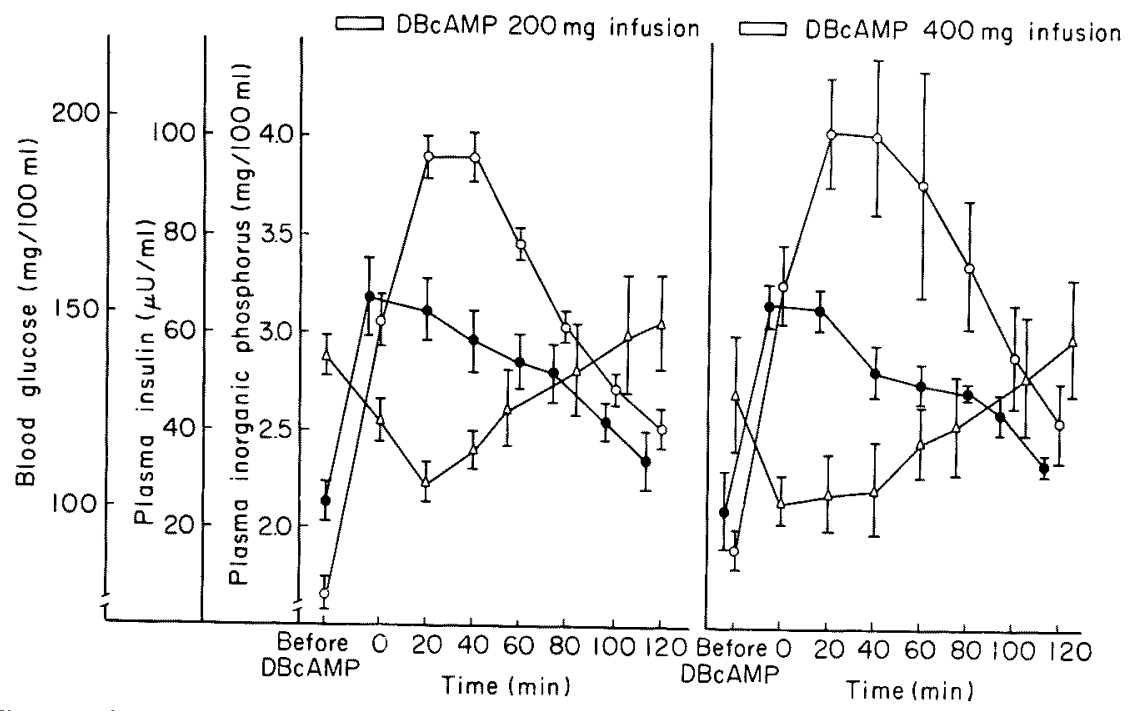

Fig. 1. Changes in blood glucose, plasma insulin and inorganic phosphorus following the administration of DBcAMP in man. Each value is expressed as mean \pm s.e. 0 , blood glucose; •, plasma insulin; $\Delta$, plasma inorganic phosphorus.

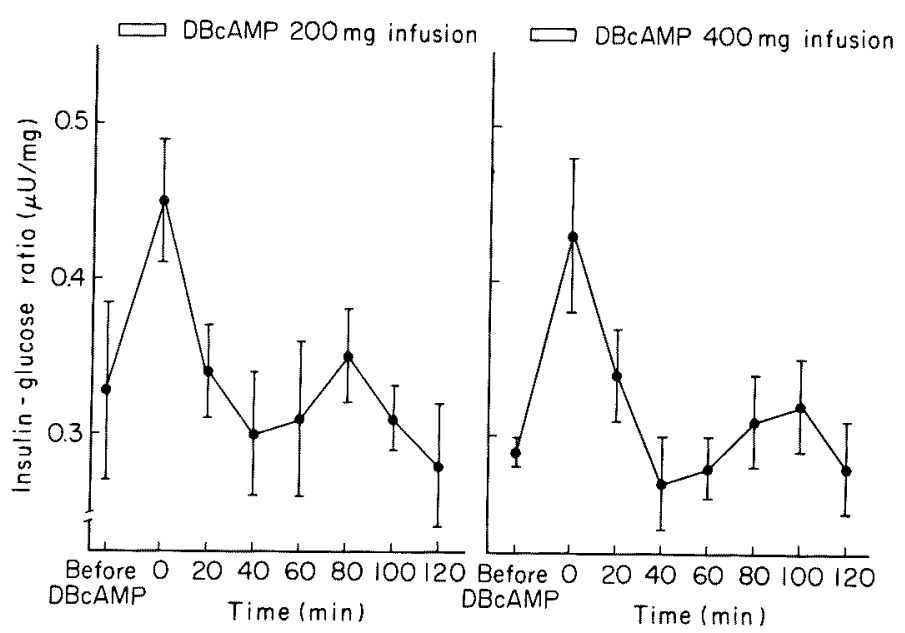

Fig. 2. Changes in I-G ratio following the administration of DBcAMP in man. Each value is expressed as mean \pm S.E.

but still remained at the elevated level even $120 \mathrm{~min}$ after infusion. The plasma IRI response curve did not correlate with that of blood glucose. Plasma Pi fell immediately reaching the minimum at about one half of its preinfusion level within $20 \mathrm{~min}$ after infusion and then returned to the preinfusion level. Insulin/glucose ratio (I/G ratio) rose significantly and reached a peak at the end of the infusion, then fell promptly even lower the preinfusion level, and elevated again before returning the initial level in both groups (Fig. 2). The blood lactate values remained 


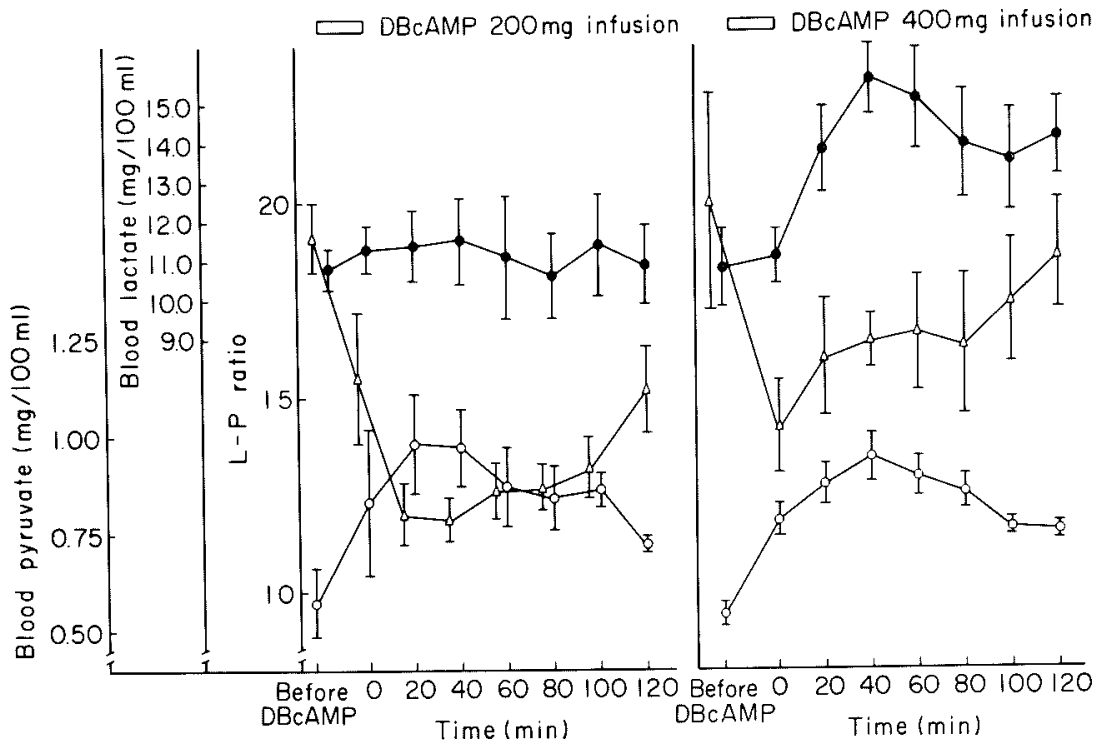

Fig. 3. Changes in blood pyruvate, lactate and L-P ratio following the administration of DBcAMPP in man. Each value is expressed as mean \pm s.e. o, blood pyruvate; • blood lactate; $\Delta$, L-P ratio.

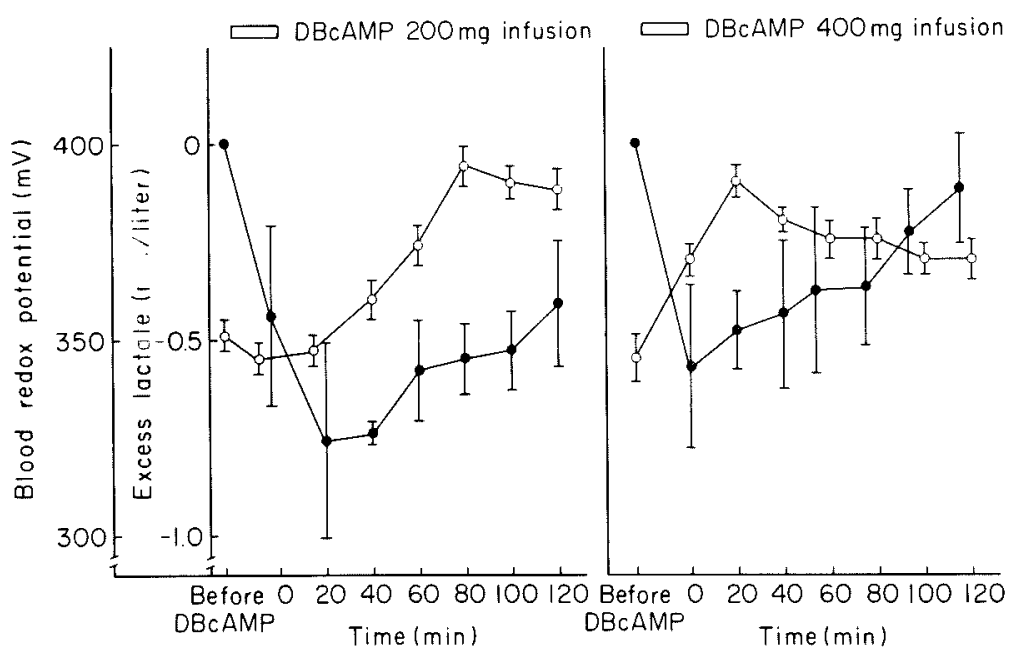

Fig. 4. Changes in blood redox-potential and excess lactate following the administration of DBcAMP in man. Each value is expressed as mean \pm s.E. o, blood redox-potential; -, excess lactate.

essentially unchanged with $200 \mathrm{mg}$ infusion, however, those values increased significantly and remained higher during a period of observation with $400 \mathrm{mg}$ infusion (Fig. 3). The blood pyruvate values increased significantly and reached a peak at $40 \mathrm{~min}$ after infusion in both groups. As a result, the lactate/pyruvate 


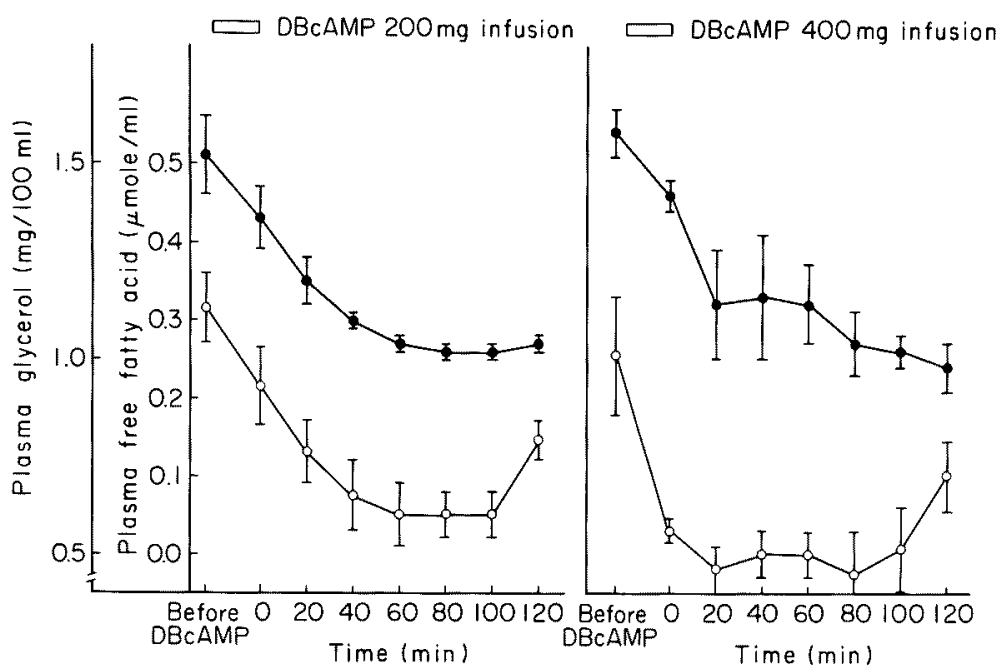

Fig. 5. Changes in plasma glycerol and nonesterified fatty acids following the administration of DBcAMP in man. Each value is expressed as mean \pm s.e. o, plasma glycerol; -, plasma free fatty acid.

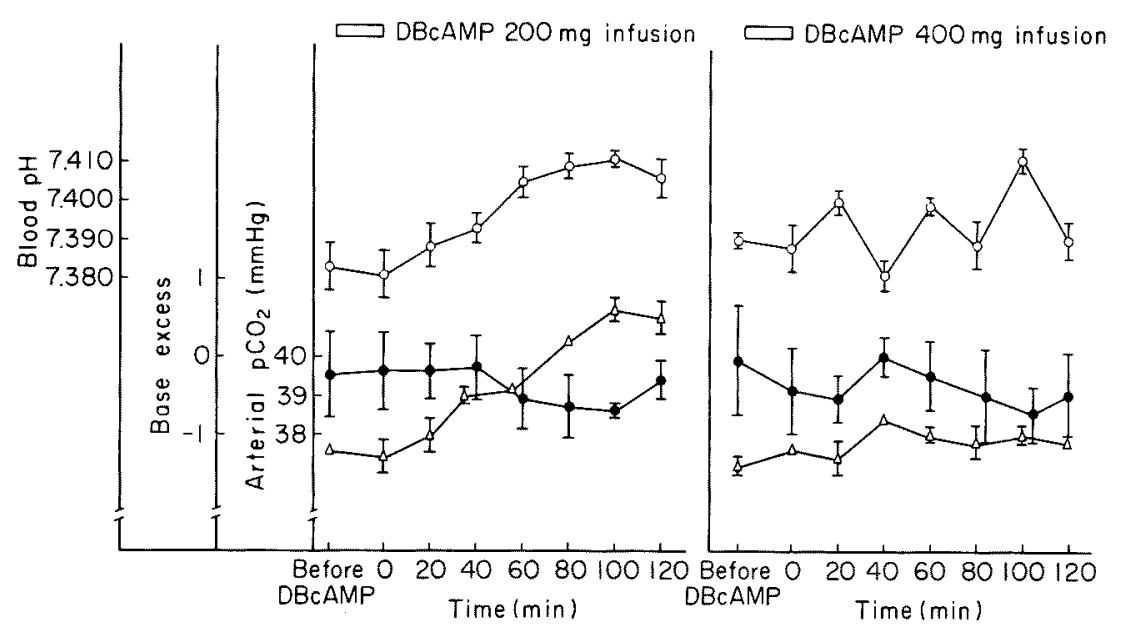

Fig. 6. Changes in blood $\mathrm{pH}, \mathrm{pCO}_{2}$ and base excess (B.E.) following the administration of DBcAMP in man. Each value is expressed as mean \pm S.E. o, blood $\mathrm{pH}$; •, base excess; $\Delta$, arterial $\mathrm{pCO}_{2}$.

ratio ( $\mathrm{L} / \mathrm{P}$ ratio) fell promptly to a lower value and remained at the low level even $120 \mathrm{~min}$ after $200 \mathrm{mg}$ infusion, but the decline in $\mathrm{L} / \mathrm{P}$ ratio was smaller with $400 \mathrm{mg}$ infusion. The pattern of change in excess lactate correlated well with the $\mathrm{L} / \mathrm{P}$ ratio pattern in both groups (Fig. 4). The elevation in the blood redoxpotential was induced by the DBcAMP infusion in both groups. The DBcAMP infusion also induced about 50 per cent decline in NEFA and glycerol in both groups (Fig. 5). The effects of DBcAMP infusion on the acid-base parameters were 


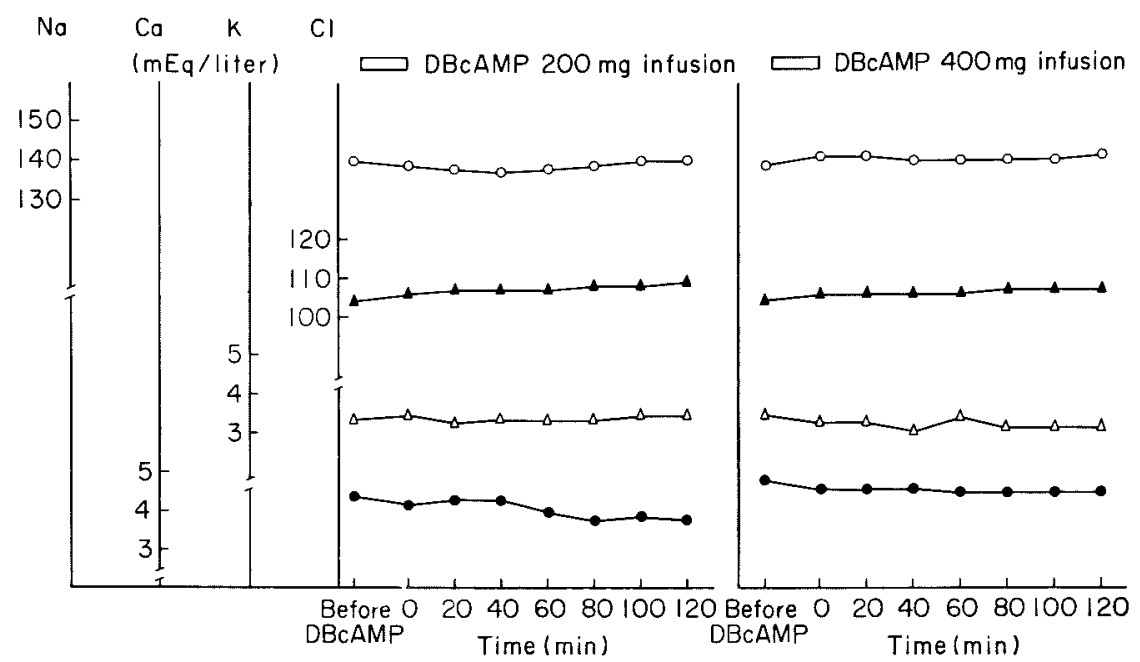

Fig. 7. Changes in serum electrolytes following the administration of DBcAMP in man.

Each value is expressed as mean \pm S.E. $\circ, \mathrm{Na} ; \bullet, \mathrm{Ca} ; \Delta, \mathrm{K} ; \bullet, \mathrm{Cl}$.

shown in Fig. 6. Arterial $\mathrm{Pa}_{\mathrm{CO}_{2}}$ remained constant because the subject's ventilation was controlled artificially during study. After $200 \mathrm{mg}$ infusion, the arterial $\mathrm{pH}$ value increased progressively, and the primary cause of that was an increase in base excess. The changes in acid-base parameters following $400 \mathrm{mg}$ infusion were less consistent. The levels of serum $\mathrm{Na}^{+}, \mathrm{K}^{+}$, and $\mathrm{Cl}-$ remained essentially unchanged in both groups. Serum $\mathrm{Ca}^{++}$levels were significantly decreased at 80 min after $200 \mathrm{mg}$ infusion, but no significant changes were observed following $400 \mathrm{mg}$ infusion.

\section{Discussion}

Sutherland and Rall (1960) originally demonstrated the role of cAMP as an activator of phosphorylase, and Posternak et al. (1962) showed that the synthetic analogue, DBcAMP, possessed similar properties. In this study, the findings that DBcAMP induced hyperglycemia which last for more than $60 \mathrm{~min}$ after infusion in human subjects, suggest that exogenous DBcAMP activates a glycogenolysis in human subjects as has been reported by Hension et al. (1967) in dogs.

DBcAMP also induced striking elevations in the plasma IRI. The elevation in the plasma IRI concentrations seemed to be induced by the direct stimulating action of DBcAMP, rather than by the glucose-stimulated insulin secretion. Though the exact mechanism for the action of DBcAMP on the insulin secretion remained unclear, it can be suggested that the increased cAMP concentration in $\beta$-cells induced by exogenous DBcAMP might play an important role in the secretion of insulin, as has been previously suggested by Heersche et al. (1971).

Significant increase in the blood pyruvate value accompanying with slightly increased blood lactate level caused by the DBcAMP infusion indicates the 
activation of glycolysis, probably as a result of the activation of phosphofructokinase, one of the key glycolytic enzymes, in a manner similar to that of cAMP as reported by Mansour and Mansour (1962). Since both pyruvate and lactate are known to penetrate readily cell membranes, the changes in $\mathrm{L} / \mathrm{P}$ ratio calculated from the blood concentrations of these metabolites suggest that of the intracellular $\mathrm{L} / \mathrm{P}$ ratios. The declines in $\mathrm{L} / \mathrm{P}$ ratios shown in Fig. 3 possibly mean the shift of the redox state of the NAD/NADH system to oxidation and elevated phosphorylation state, i.e. $[\mathrm{ATP}] /[\mathrm{ADP}][\mathrm{Pi}]$, in cytoplasm (Krebs 1967). This is supported by the elevation in blood redox-potential, depression in the $\mathrm{Pi}$ concentration and metabolic alkalosis following DBcAMP infusion. These changes induced by the DBcAMP infusion might be ascribed to an enhanced flux of hydrogen equivalent from cytoplasm into mitochondria via the "glycerol phosphate cycle" as considered by Müllhofer et al. (1974).

The infusion of $200 \mathrm{mg}$ and $400 \mathrm{mg}$ of DBcAMP into human subjects produced progressive and parallel declines in NEFA and glycerol concentrations in plasma for more than $2 \mathrm{hr}$ after infusion. Blecher (1967) observed in isolated rat epididymal fat cells that a higher concentration of DBcAMP stimulated lipolysis while a lower concentration of DBcAMP inhibited. Infusion of DBcAMP at a rate of $10-20 \mathrm{mg} / \mathrm{min}$ for $20 \mathrm{~min}$ probably inhibited lipolysis.

From these results, it is concluded that DBcAMP in dose ranging from 200 to $400 \mathrm{mg}$ stimulates glycogenolysis and glycolysis while lipolysis is inhibited.

\section{Acknowledgment}

I am indebted to Daiichi Pharmaceutical Co., Ltd. Japan for their gift of DBcAMP. The excellent technical assistance of Miss Junko Miyao and Miss Teruko Sameshima is gratefully acknowledged. I wish to express my gratitude to Prof. Koroku Hashimoto, Department of pharmacology, Tohoku university, and Prof. Junichi Yoshitake for many valuable suggestions and discussions of this work.

\section{References}

1) Astrup, P. (1957) A simple electric technique for the determination of carbon dioxide tension in blood and plasma, total content of carbon dioxide in plasma and bicarbonate content in separated plasma at fixed carbon dioxide tension (40 $\mathrm{mmHg}$ ). Scand. J. clin. Lab. Invest., 8, 33-49.

2) Bergmeyer, H.U. (1963) Methods of Enzymatic Analysis. Academic Press. New York and London.

3) Blecher, M. (1967) Evidence for the involvement of cyclic-3',5'-adenosine monophosphate in glucose utilization by isolated rat epididymal adipose cells. Biochem. Biophys. Res. Commun., 27, 560-567.

4) Fiske, C.H. \& Subbarow, T. (1925) The colorimetric determination of phosphorus. J. biol. Chem., 66, 375, Cited. by I. Kanai, Rinsho Kensa no Teiyo (Jap.), 1964.

5) Fujii, S. \& Okuda, H. (1972), Role of cyclic AMP in fat mobilization. Taishia (Jap.), 9, 754-759.

6) Hales, C.N. \& Randle, P.J. (1963), Immunoassay of insulin with insulin-antibody precipitate. Biochem. J., 88, 137-146.

7) Heersche, J.N., Fedak, S.A. \& Aurbach, G.D. (1971) The mode of action of dibutyryl adenosine 3', 5'-monophosphate on bone tissue in vitro. J. biol. Chem., 246, 67706775 . 
8) Henion, W.F., Sutherland, E.W. \& Posternak, T.H. (1967) Effects of derivatives of adenosine $3^{\prime}, 5^{\prime}$-phosphate on liver slices and intact animals. Biochem. Biophys. Acta, 148, 106-113.

9) Krebs, H.A. (1967) The redox state of nicotinamide-adenine dinucleotide in the cytoplasm and mitochondria of rat liver. Advances in Enzyme Regulation, 5, 409-434.

10) Levine, R.A., Levis, S.E., Shulman, J. \& Washington, A. (1969) Metabolism of cyclic adenosine 3 ',5'-monophosphate-8-1 ${ }^{14} \mathrm{C}$ by isolated, perfused rat liver. $J$. biol. Chem., 244, 4017-4022.

11) Mansour, T.E. \& Mansour, J.M. (1962) Effects of serotonine (5-hydroxytryptamine) and adenosine 3',5'-phosphate on phosphofructokinase from the liver fluke fasciola hepatica. J. biol. Chem., 237, 629-634.

12) Müllhofer, G., Loy, E., Wollenberg, P. \& Krämer, R. (1974) A possible role of the glycerol phosphate cycle in cyclic AMP-stimulated gluconeogenesis from lactate in perfused rat livers. Physiol. Chem., 355, 239-254.

13) Posternak, T.H., Sutherland, E.W. \& Henion, W.F. (1962) Derivatives of cyclic 3', $5^{\prime}$-adenosine monophosphate. Biochim. Biophys. Acta, 65, 558-560.

14) Sutherland, E.W. \& Rall, T.W. (1958) Fractionation and characterization of a cyclic adenine ribonucleotide formed by tissue particles. $J$. biol. Chem., 232, 1077-1091.

15) Sutherland, E.W. \& Rall, T.W. (1960) The relation of adenosine $3^{\prime}, 5^{\prime}$-phosphate and phosphorylase to the actions of catecholamines and other hormones. Pharmacol. Rev., 12, 265-299.

16) Yoshimura, N., Kodama, K. \& Yoshitake, J. (1971) Carbohydrate metabolism and insulin release during ether and halothane anaesthesia. Brit. J. Anaesth., 43, 1022-1026. 